\title{
Insulin-like growth factor-1 in articular cartilage repair for osteoarthritis treatment
}

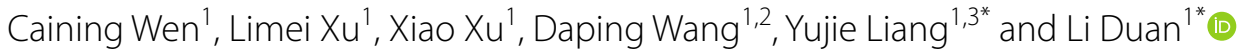

\begin{abstract}
Articular cartilage repair is a critical issue in osteoarthritis (OA) treatment. The insulin-like growth factor (IGF) signaling pathway has been implicated in articular cartilage repair. IGF-1 is a member of a family of growth factors that are structurally closely related to pro-insulin and can promote chondrocyte proliferation, enhance matrix production, and inhibit chondrocyte apoptosis. Here, we reviewed the role of IGF-1 in cartilage anabolism and catabolism. Moreover, we discussed the potential role of IGF-1 in OA treatment. Of note, we summarized the recent progress on IGF delivery systems. Optimization of IGF delivery systems will facilitate treatment application in cartilage repair and improve OA treatment efficacy.
\end{abstract}

Keywords: IGF-1, Cartilage repair, Chondrocytes, Osteoarthritis

\section{Introduction}

Osteoarthritis (OA) is characterized by the progressive destruction of articular cartilage, which seriously restricts sports ability and impacts quality of life. The primary function of articular cartilage is to reduce the friction between joints and make joint movement smooth, soft, and painless [1]. Articular cartilage is mainly composed of chondrocytes and dense extracellular matrix (ECM) without blood vessels or innervation. Thus, chondrocyte metabolism in the adjacent cartilage is relatively low, and these cells cannot easily migrate to the damaged site [2]. Once a cartilage defect occurs, self-repair is not easy [3].

Currently, the typical treatment for the early stage of OA is anti-inflammatory drugs and hyaluronic acid injection. These conservative strategies can alleviate pain symptoms, but they cannot terminate the progression

\footnotetext{
*Correspondence: liangyjie@126.com; duanl@szu.edu.cn

1 Department of Orthopedics, Guangdong Provincial Research Center for Artificial Intelligence and Digital Orthopedic Technology, Shenzhen Second People's Hospital, The First Affiliated Hospital of Shenzhen University, Shenzhen 518035, China

${ }^{3}$ Department of Child and Adolescent Psychiatry, Shenzhen Kangning Hospital, Shenzhen Mental Health Center, Shenzhen 518003, China

Full list of author information is available at the end of the article
}

of cartilage deterioration and repair cartilage defects. Alternative therapies, including autologous chondrocyte implantation (ACI), matrix-induced ACI (MACI), and stem cell transplantation, are also available commercially or are in the clinical study phase. However, clinical efficacy of these treatments is not conclusive, as these treatments do not consistently regenerate hyaline cartilage. Finally, clinicians can use surgical methods such as joint replacement for the late-stage OA, which is a substantial economic and physical to patients and society. In addition to a series of risks during and after the joint replacement operation, total knee replacement surgery occurs in as many as $5 \%$ of cases, requiring revision surgery ten years after the operation [4]. Strategies to optimize OA treatment outcomes are urgently needed.

Growth factors and their signaling pathways have recently attracted much attention in cartilage repair for OA treatment. Insulin-like growth factor-1 (IGF-1), a member of a family of growth factors that are structurally closely related to pro-insulin, has shown profound effects on chondrocyte biological behavior and fundamentally regulates cartilage matrix metabolism during cartilage repair. Strategies for IGF delivery to chondrocytes and cartilage matrix are essential for its clinical application in OA treatment.

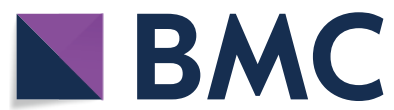

(c) The Author(s) 2021. Open Access This article is licensed under a Creative Commons Attribution 4.0 International License, which permits use, sharing, adaptation, distribution and reproduction in any medium or format, as long as you give appropriate credit to the original author(s) and the source, provide a link to the Creative Commons licence, and indicate if changes were made. The images or other third party material in this article are included in the article's Creative Commons licence, unless indicated otherwise in a credit line to the material. If material is not included in the article's Creative Commons licence and your intended use is not permitted by statutory regulation or exceeds the permitted use, you will need to obtain permission directly from the copyright holder. To view a copy of this licence, visit http://creativecommons.org/licenses/by/4.0/. The Creative Commons Public Domain Dedication waiver (http://creativeco mmons.org/publicdomain/zero/1.0/) applies to the data made available in this article, unless otherwise stated in a credit line to the data. 


\section{IGF-1 in cartilage metabolism}

\section{IGF-1 promotes cartilage anabolism}

Proteoglycan is an essential component of the ECM. An ex vivo study showed that IGF-1 in fetal bovine serum was responsible for maintaining articular cartilage proteoglycan synthesis. Thus, IGF has great potential to promote the regeneration of articular cartilage after injur $y[5]$. Another study has demonstrated that both serum and synovial fluid from rheumatoid arthritis (RA) could not stimulate chondrocyte proteoglycan synthesis once the IGF-1 function was blocked by a primary antibody [6]. Therefore, IGF-1 is the crucial factor in serum and synovial fluid that promotes cartilage matrix anabolism. Besides stimulating ECM production, IGF-1 can stimulate the proliferation and chondrogenic differentiation of mesenchymal stem cells (MSCs) [7].

In vivo experiments have similar results. In a rat fracture model, IGF-1 combined with TGF- $\beta$ could stimulate chondrocyte proliferation and cartilage formation at the early stage of day 5 [8]. IGF directly stimulates the proliferation of chondrocytes derived from rabbit ears, costal cartilage, articular joints, and growth plates $[9,10]$. The presence of IGF-1 is crucial for maintaining cartilage integrity. When cartilage is damaged, MSCs derived from synovial fluid can partially move to the injured site and differentiate into chondrocytes to repair the defect, and IGF-1 induces chondrogenic differentiation [11-13]. An animal study in rats has confirmed that a continuous decrease in IGF-1 results in cartilage damage [14]. In addition, IGF-1 can stimulate the synthesis of type II collagen [15]. MSCs infected with the adenoviral (Ad) vector AdIGF-1 were fixed in fibrin glue and transferred to damaged rat cartilage. These cells could produce type II collagen and induce satisfactory repair results that were rich in protein aggregates [16]. In the sugar control group, the damaged area was empty or filled with fibrocartilage, composed of type I collagen. A further study [17] was performed to investigate the role of HIF-1 $\alpha$ in cartilage regeneration using adeno-associated virus (AAV) and Ad vectors to overexpress IGF-1 in the knee joint of miniature pigs. Then, autologous periosteal cells combined with a scaffold overexpressing IGF-1 were implanted in the cartilage defect. The results showed that in the group that was administered periosteal cells without IGF-1 overexpression, a fibrous ECM was detected in the superficial layer of the defect, and there was little HIF- $1 \alpha$ staining. Fibrocartilage matrix forms were identified in the deep layer and exhibited moderate HIF- $1 \alpha$ expression. In contrast, periosteal cells with Ad/AAVinduced IGF-1 expression maintained a chondrocytelike phenotype and produced a hyaline-like matrix with intense intracellular HIF- $1 \alpha$ staining in the regenerated tissue area. This study indicates that IGF-1 can increase the expression of HIF- $1 \alpha$ and that a simulated hypoxic environment is favorable for the regeneration of precursor cells in cartilage.

IGF-1 promotes MSC chondrogenic differentiation and chondrocyte proliferation mainly through the activation of IGF-1R. The primary substrates recruited after IGF-1R activation are the insulin receptor-substrate (IRS) family members IRS-1 and IRS-2. Once IRS is phosphorylated, downstream signaling pathways are activated, including the phosphoinositide 3-kinase (PI3K) cascade and extracellular signal-regulated kinase (ERK). ERK is a member of the mitogen-activated protein kinase (MAPK) cascade [18]. IGF-1 activates PI3K through the IGF-1 tyrosine kinase receptor and activates the downstream target kinase Akt, thus activating mammalian target of rapamycin (mTOR). mTOR activation can induce chondrocyte proliferation and differentiatio $\mathrm{n}[11,19]$. The Ras and Raf/ MAPK/ERK (MEK) cascades are also activated during IGF-1-induced chondrocyte proliferation and differentiation. Figure 1 shows a schematic diagram of the IGF-1/PI3K/Akt and IGF-1/MAPK/ERK signaling pathways. An in-depth investigation suggested that IGF-1 stimulates human articular chondrocytes to synthesize proteoglycans by activating the PI3K/Akt/mTOR signaling pathway without activating the Ras/Raf/MEK/ERK pathway [18]. Additionally, IGF-1 may be associated with a low oxygen microenvironment since IGF-1 stimulates the production of hypoxia inducible factor- $1 \alpha$ (HIF-1 $\alpha$ ) protein through the PI3K/mTOR and MEK/ERK pathways [20-22]. The essential role of HIF-1 $\alpha$ has been recognized in joint cartilage formation and chondrocyte phenotype maintenance during development [23-25].

\section{IGF-1 inhibits cartilage catabolism}

When OA occurs, the ECM, especially type II collagen and aggrecan, is degraded. OA pathology is reversible in the early stage but irreversible at the late stage. It is well recognized that cartilage matrix degradation is stimulated by inflammatory factors, such as tumor necrosis factor-alpha (TNF- $\alpha$ ) and interleukins (IL-1 and IL-7), and the production of matrix metalloproteinase-13 (MMP-13), which can degrade the major components of the ECM $[26,27]$.

Many studies have confirmed that IGF-1 can reduce the metabolism of the cartilage matrix. Amanda et al. found that adding IL-1 to cartilage explant cultures could degrade proteoglycans in the cartilage, and chondrocytes can take up IL-1 for further degradation. The addition of IGF-1 inhibits IL-1-induced matrix degradation [28]. IGF-1 inhibits IL- $1 \beta$-induced NF- $\mathrm{kB}$ activation by inhibiting IкB- $\alpha$ kinase. NF-kB-regulated gene products are extensively involved in inflammation and cartilage degradation (COX-2, MMPs) and apoptosis (caspase-3). The 


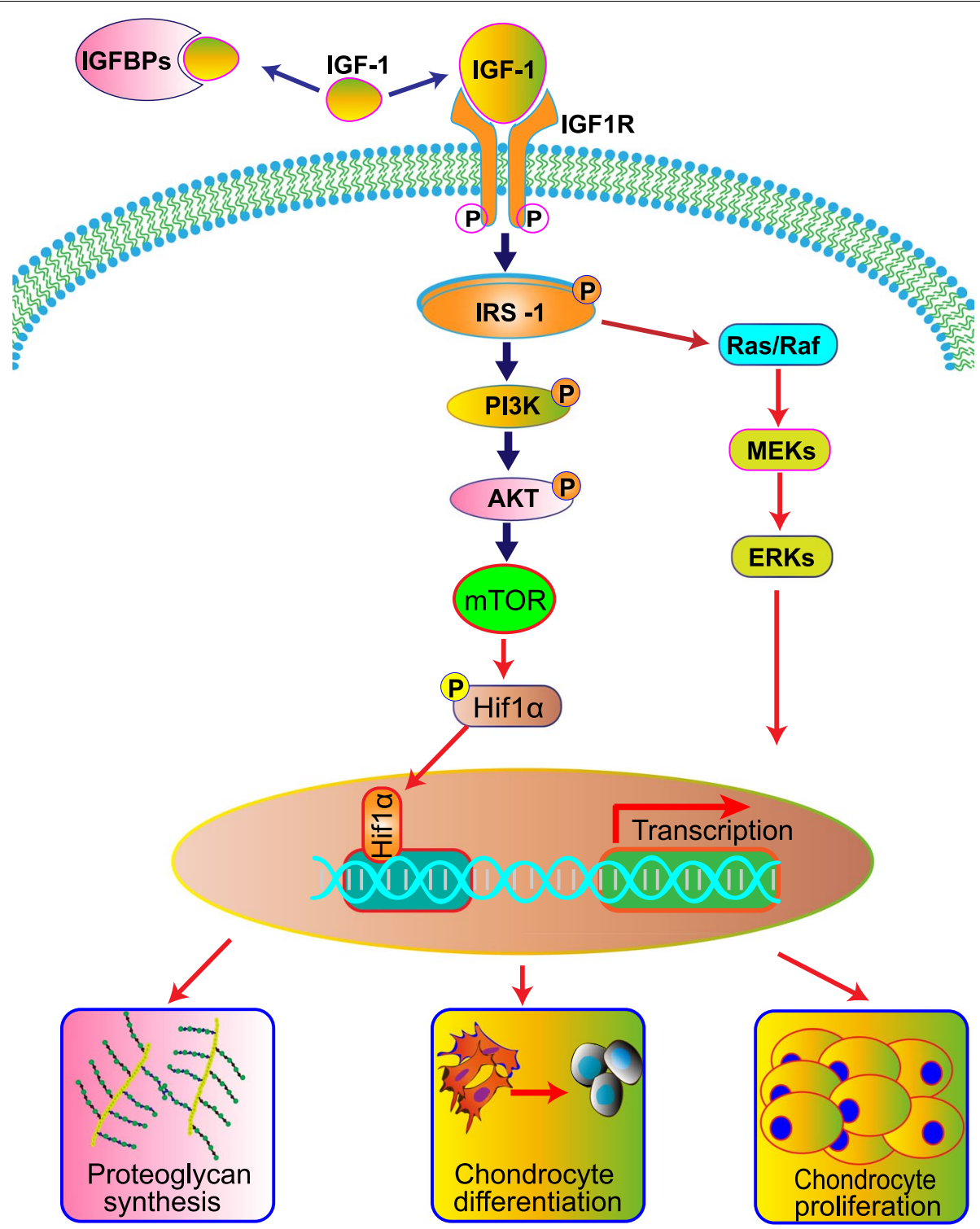

Fig. 1 Schematic diagram of the IGF-I/PI3K/Akt and IGF-I/MAPK/ERK signaling pathways in articular cartilage metabolism. IGF-1 contributes to the chondrogenic differentiation of mesenchymal stem cells (MSCs). IGF-1 promotes proteoglycan synthesis in chondrocytes by activating the PI3K pathway and stimulates chondrocyte proliferation via the PI3K and MEK/ERK pathways

inhibitory effects of IGF-1 on IL-1 $\beta$-induced NF-kB activation are partially mediated by inhibiting the Src/PI3K/ AKT pathway [29]. In addition, in rat endplate chondrocytes, the intervention of IGF-1 up-regulated the expression of MMP-13 through the ERK pathway, thereby inhibiting the decomposition of cartilage [30].

Chondrocyte apoptosis is a typical characteristic of $\mathrm{OA}$. One of the IGF-1 functions is to protect chondrocytes from apoptosis [31, 32]. In a study by Higgins et al., IGF-1 was used to treat medial femoral condylar fractures in New Zealand white rabbits. Compared to the control group, the fibrin clot containing IGF-1 $(25 \mu \mathrm{g} /$ $\mathrm{mL}$ ) for fracture repair inhibited chondrocyte apoptosis in vivo [33].

\section{IGF-1 in OA progression}

In addition to its effects on cartilage metabolism, IGF-1 affects the development of OA in other ways. The addition of IGF-1 led to the repair of cartilage and the repair of subchondral bone [34]. In another study, researchers explored the effects of different doses of IGF-1 on cartilage and subchondral bone repair. They found that 
high-dose IGF-1 was beneficial to cartilage formation, while low-dose IGF-1 was more conducive to subchondral bone reconstruction [35]. As mentioned above, IGF-1 can inhibit the production of IL-1 and metalloproteinase. Therefore, we can infer IGF-1 could alleviate synovitis with reduced inflammation. Furthermore, the ability of IGF-1 to reduce synovial inflammation has been proved in an equine model [36].

\section{The risks of IGF-1 application}

The relationship between IGF-1 and cellular senescence is still unclear and controversial. Some results suggested that IGF-1 could delay cartilage aging while some studies demonstrated that IGF-1 could accelerate the aging process of cartilage. In aging and dedifferentiated chondrocytes, researchers found that IGF-1 expression level was downregulated. In contrast, the aging factor was upregulated, suggesting that reducing IGF-1 may result in the aging of chondrocytes [37]. Another study indicates that autocrine production of IGFs contributes to maintaining the survival of chondrocytes in vitro and in vivo [38]. However, the ability of IGF-1 to stimulate proteoglycan synthesis in chondrocyte decreases with the age of the donor increasing [39]. In another study on monkeys, the response of cartilage samples to IGF-1 significantly reduced with aging [40]. The mechanism may be related to the decrease of forkhead-box class O (FOXO) transcription factor expression in chondrocytes, thus weakening the cell's resistance to oxidative stress [41]. Then, oxidative stress inhibits IGF-1 to stimulate Akt phosphorylation and increases ERK phosphorylation, thereby inhibiting cartilage synthesis [39]. Controversial evidence suggested that IGF-1 could accelerate the aging of chondrocytes. Zhao et al. [42] found that IGF-1 promoted the senescence of rat articular chondrocytes by activating senescence-associated $\beta$-galactosidase (SA- $\beta$-gal) and up-regulating p53 and p21 expression levels. Thus, the interaction between IGF-1 and chondrocyte senescence need further investigation. Furthermore, whether IGF1 will lead to the excess production of cartilage, such as ectopic cartilage, is still a vacant field that we may make efforts.

\section{IGF-1 in osteoarthritis treatment}

Studies have shown that articular cartilage isolated from arthritic mice lacks a response to IGF-1 [43]. The reason may be that chondrocytes in OA produce excessive IGF receptor-binding protein, which blocks the activation of IGF receptors [44]. Thus, when using IGF-1 to treat OA, a simple injection of IGF-1 into the damaged site may not achieve a therapeutic effect.

Currently, most OA treatment with IGF-1 is based on tissue engineering. Cartilage tissue engineering involves seeding cells on a scaffold to support tissue growth and phenotypic maintenance by regulating growth factors and producing engineered cartilage with structures and functions equivalent to those of natural tissue $[45,46]$. Tissue engineering combined with gene therapy is promising for articular cartilage repair. The transfection of articular chondrocytes with IGF-1 cDNA enhances the structural and functional properties of tissue-engineered cartilage based on polyglycolic acid scaffolds [47]. In another study [48], chondrocytes overexpressed IGF-1, and these genetically modified chondrocytes were cultured on a polyglycolic acid scaffold in vitro. The cartilage construct was implanted into the osteochondral defect of the knee joint in rabbits. After 28 weeks, the construct was analyzed. Compared to constructs without IGF-1 overexpression, IGF-1-overexpressing constructs significantly promoted osteochondral repair and reduced cartilage degeneration near the defect. IGF-1 delivery relies on tissue engineering methods, as shown in Fig. 2. Some studies on the application of IGF-1 via tissue engineering are shown in Table 1.

Periosteum could be a very suitable tissue graft because it meets the three main tissue engineering requirements: seeding cells, a scaffold, and regulatory growth factors. Periosteum was transplanted into a defect and then developed into cartilage under the regulation of growth factors [52]. Matsumoto et al. [53] showed that cells in periosteal explants stimulated with IGF-1 $(100 \mathrm{ng} / \mathrm{mL})$ for two days differentiated into chondrocytes. Prolonged incubation (e.g., 6 weeks) of periosteal explants in serumfree medium led to type II collagen expression.

\section{IGF-1 in clinical trials}

The effect of IGF-1 on OA treatment has been extensively confirmed in vitro studies. However, the clinical evidence of IGF-1 in OA treatment is minimal. In prospective clinical trials (study number DRKS00000365 and UKF001822), by analyzing the growth factor content in the synovial fluid of patients with OA, they found the IGF-1 concentration in the joints with cartilage lesions was significantly increased compared to healthy samples [54]. The increase of local IGF-1 binding protein prevents IGF-1 from freely exerting its biological effects [55]. Therefore, IGF-1 delivery strategies for OA treatment should be optimized. In addition, IGF-1 based-target therapies are under consideration to avoid its side effects.

\section{Strategies for IGF-1 delivery}

IGF-1, as a small and highly diffusible protein, can be transported long distances to exert its biological effects [56]. However, as a molecule with close structural homology to insulin, IGF-1 elicits a variety of metabolic products in vitro and in vivo that are similar to insulin, which 

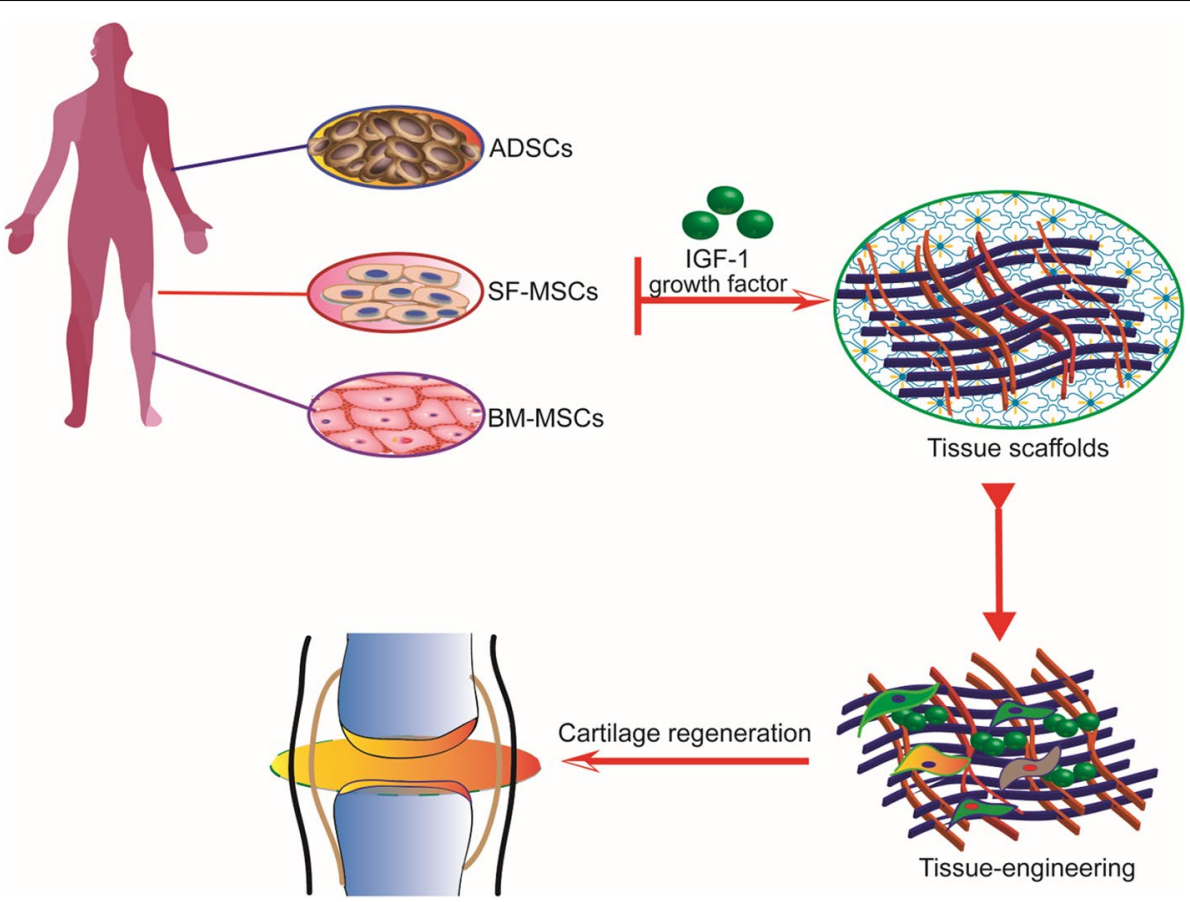

Cartilage regeneration

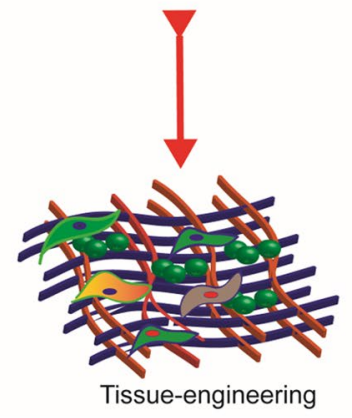

Fig. 2 Tissue engineering-based IGF-1 delivery. MSCs, such as ADSCS, SF-MSCs, and BM-MSCs, are seeded on scaffolds. The addition of IGF-1 can induce chondrogenic differentiation of MSCs and promote cartilage tissue formation, cartilage matrix GAG accumulation, and type II collagen production, thus promoting cartilage regeneration and cartilage defect repair

Table 1 Tissue engineering-based applications of IGF-1

\begin{tabular}{|c|c|c|c|c|}
\hline Growth factors & Scaffolds & Cells & Effect & References \\
\hline IGF-1/BMP-2 & Fibrin glue & $\begin{array}{l}\text { Mesenchymal cells isolated } \\
\text { from rib perichondrium }\end{array}$ & $\begin{array}{l}\text { Infecting mesenchymal stem cells with the } \\
\text { adenoviral vectors AdBMP-2 and AdlGF-1 } \\
\text { to induce transparent cartilage repair }\end{array}$ & {$[16]$} \\
\hline IGF-1/TGF- $\beta$ & Polyglycolic acid (PGA) scaffolds & Chondrocytes & $\begin{array}{l}\text { The presence of IGF-1/TGF- } \beta \text {,or genetically } \\
\text { modified chondrocytes overexpressing } \\
\text { IGF-1 significantly improved osteochondral } \\
\text { repair }\end{array}$ & {$[46,48]$} \\
\hline |GF-1 & $\begin{array}{l}\text { Type Il collagen glycosaminoglycan (CG) } \\
\text { scaffold }\end{array}$ & Adult articular chondrocytes & $\begin{array}{l}\text { Continuous local overexpression of IGF-1 } \\
\text { leads to enhanced cartilage formation }\end{array}$ & [49] \\
\hline IGF-1/BMP-2 & $\begin{array}{l}\text { Bilayered oligo (poly(ethylene glycol) fuma- } \\
\text { rate) (OPF) hydrogel composites }\end{array}$ & Mesenchymal stem cells & $\begin{array}{l}\text { IGF-1 and BMP-2 can synergistically } \\
\text { enhance the formation of subchondral } \\
\text { bone, and the delivery of IGF-1 alone has a } \\
\text { positive effect on the repair of osteochon- } \\
\text { dral tissue }\end{array}$ & {$[50,51]$} \\
\hline
\end{tabular}

can cause edema primarily in the face and hands, mild weight gain, occasional dyspnea, bilateral jaw tenderness, arthralgias and myalgias, fatigue, tachycardia, flushing, orthostatic, and hypotension [57]. These long-term side effects and the risks of carcinogenesis [58] seriously compromise the systemic application of IGF-1. The biggest obstacle regarding local delivery is inflammation-induced IGF-1 degradation [59]. Therefore, considerable efforts have been made to improve IGF-1 delivery technologies.

\section{Gene delivery}

In vitro and in vivo studies have proven the efficacy of IGF-1 gene therapy in OA treatment [16, 60, 61]. Viral and nonviral vectors have been used for IGF-1 gene delivery. The advantages and disadvantages of both systems are described in Table 2.

At present, viral vector-mediated gene transfer of IGF-1 has been widely documented. Kyla et al. [67] showed that chondrocytes from horses that were transduced with 
Table 2 The pros and cons of different genetic methods for IGF-1 delivery

\begin{tabular}{|c|c|c|c|}
\hline Vectors & Advantage & Disadvantage & References \\
\hline Retroviral vector & Long-term expression of genes incorporated into host cells & $\begin{array}{l}\text { Insertion mutations, difficult to terminate } \\
\text { expression, premature senescence of cells }\end{array}$ & {$[1,62]$} \\
\hline Adenoviral vector & High transfection efficiency and high level of gene products & Severe immunogenicity & {$[63]$} \\
\hline Adeno-associated virus vector & $\begin{array}{l}\text { Excellent long-term gene transfer efficiency, no immune } \\
\text { response, and a lack of toxicity and mutagenesis }\end{array}$ & Small target genes can be inserted & {$[64,65]$} \\
\hline Lentiviral vector & $\begin{array}{l}\text { Larger cDNA can be introduced into target cells without } \\
\text { causing immune responses }\end{array}$ & Safety unknown & [66] \\
\hline Nonviral gene delivery & Improved safety & The long-term effect is not good & [1] \\
\hline
\end{tabular}

rAAV5-IGF-1 exhibited improved histological scores, including increased chondrocyte predominance and collagen type II production. Ad vectors are the most popular viral vectors and have high transfection efficiency. In one study [16], AdIGF-1-infected MSCs were fixed in fibrin glue and implanted in rat cartilage defects, which led to satisfactory cartilage repair (type II collagen matrix secretion and hyaline cartilage production). However, Ad vectors may trigger severe host reactions, including causing patient death during the first phase of gene therapy clinical trials [63]. Therefore, more substantial evidence is still needed to support the clinical application of Ad vectors.

Nonviral gene delivery refers to DNA passage through cell membranes using physical and chemical methods such as electroporation. Nonviral gene delivery systems are advantageous compared to virus transfection because these methods can avoid mutations caused by retroviral gene transfection and reduce the immune response. Moreover, the cargo is not inserted into the target cell genome. Biological safety guarantees the application of this delivery system in chronic disease treatment that requires long-term export of gene products [1]. A scaffold made up of type II collagen and glycosaminoglycan have been used as a nonviral gene delivery vector. The scaffold was soaked in an IGF-1 plasmid solution with a Lipofection reagent. Rapid release and sustained IGF-1 overexpression resulted in significant glycosaminoglycan accumulation and type II collagen production [49].

\section{Protein delivery}

In addition to gene delivery, various IGF-1 protein delivery systems have been developed. Tokunou et al. [68] designed and purified a new protein named heparin bound to IGF-1 (Xp-HB-IGF-1). The researchers found that Xp-HB-IGF-1 was selectively retained by cartilage explants and led to continuous chondrocyte proteoglycan biosynthesis. Julian C. Lui et al. [69] developed a cartilage-targeted single-chain human antibody fragment $(\mathrm{CaAb})$ and fused this fragment to IGF-1. The
CaAb-IGF-1 fusion protein had sustained cartilage binding ability and IGF-1 biological activity, promoting cartilage formation in model mice.

In addition, small cationic nanocarriers $(<15 \mathrm{~nm})$ can overcome the biological barrier of joints by binding and penetrating anionic cartilage tissue. Studies have shown that the penetration speed of these nanocarriers was faster than the rate of carrier clearance by the joint space [70, 71]. IGF-1 was loaded into amine-terminated polyamide-amine dendrimer nanocarriers, and then different polyethylene glycol concentrations were used to control the surface charge to enhance local function [72]. The tissue uptake formula increased the residence time of therapeutic IGF-1 in the rat knee by ten times and showed improved cartilage repair. This study demonstrated that the nanocarrier could improve the pharmacokinetics and clinical efficacy of IGF-1 for OA treatment.

Steven $\mathrm{Lu}$ et al. [50] made a biodegradable hydrogel composite scaffold with macromonomer oligo (polyethylene glycol) fumarate (OPF), which simulated the structural layer of the osteochondral unit. IGF-1 and bone morphogenetic protein 2 (BMP-2) were loaded into gelatin particles. The study results showed that the double-layer OPF hydrogel complex had a robust spatial orientation for cartilage tissue repair and tremendous growth factor release potential. IGF-1 and BMP-2 synergistically promoted subchondral bone formation. Figure 3 shows that the delivery of IGF-1 protein to each layer of cartilage plays a therapeutic role.

Moreover, tissue or cell-specific delivery of the gene vectors by designed carriers enables an advanced version of gene therapy with reduced toxicity or risk [73]. In recent years, great progress has been made in engineered exosomes based on genetic modification. Exosome engineering using genetic and chemical methods for targeted drug delivery boasts low toxicity, low immunogenicity, and high engineerability, which holds promise for cellfree therapies [74]. Liang et al. [75] showed a genetic engineering strategy to modify exosome surfaces, achieve excellent chondrocyte-specific targeting and cartilage 


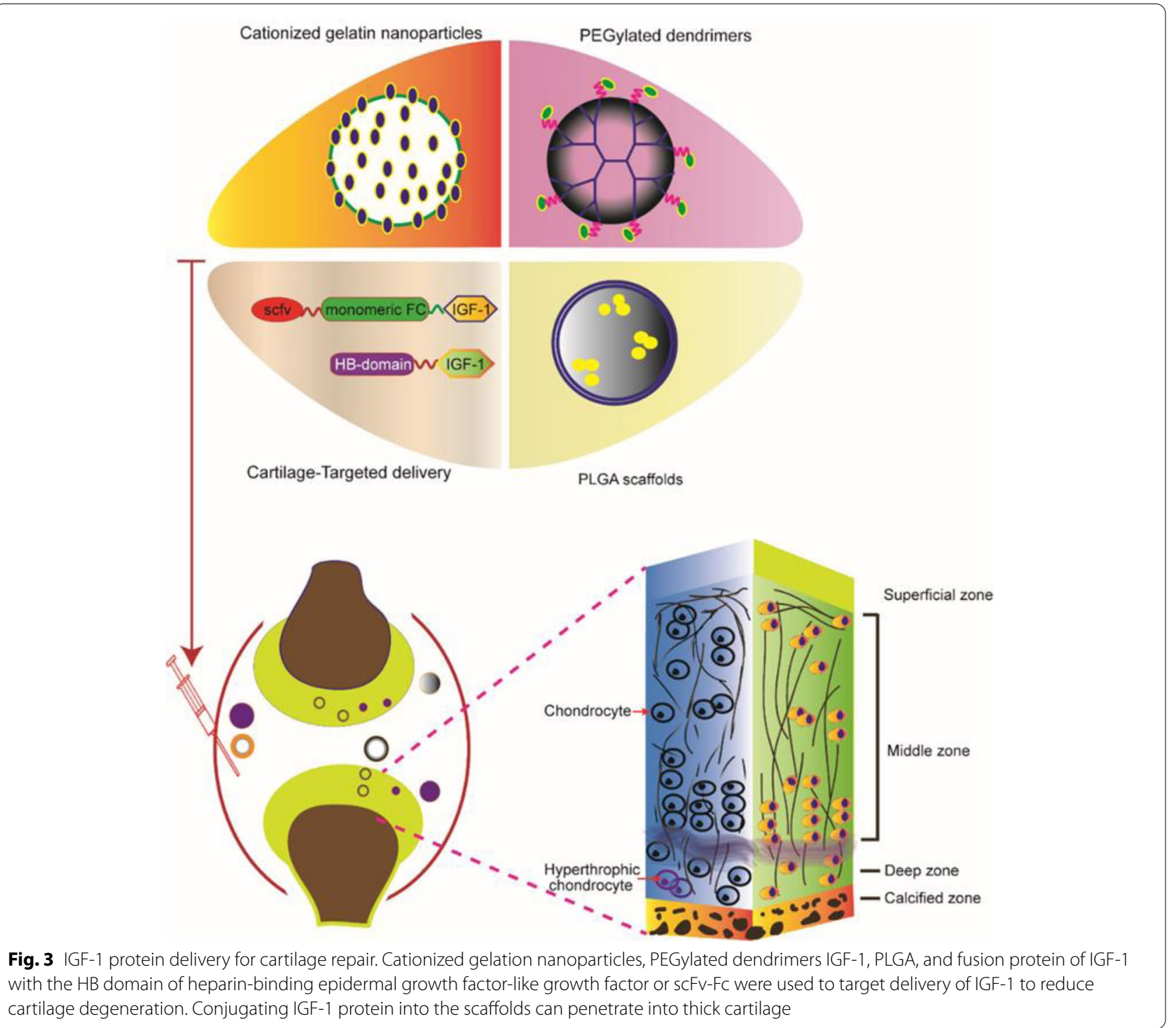

penetration, and rejuvenate the OA cartilage. $\mathrm{Xu}$ et al. [76] reported that targeted delivery of kartogenin (KGN) to synovial fluid MSCs (SF-MSCs) by engineered exosomes leads to even dispersion of KGN in the cytosol and strongly promotes the chondrogenesis of SF-MSCs in vitro and in vivo. These suggest that the delivery of IGF-1 through an engineered exosome system is a valuable research direction.

\section{Conclusion}

IGF-1 profoundly regulates cartilage repair by promoting cartilage anabolism and inhibiting cartilage catabolism. Besides, IGF-1 may delay OA progression. Effective delivery of IGF-1 to injured cartilage is a prerequisite for growth factors to exert biological functions in vivo.
Current IGF-1 delivery technologies based on viral or nonviral systems need further optimization. Delivery of IGF-1 through engineered exosomes may become an efficient way in the future. However, more in-depth studies should be performed to analyze IGF function and safety before its clinical application. In summary, with the advancement and optimization of delivery systems, IGF-1 will become an alternative cartilage repair strategy for OA treatment in the future.

\section{Abbreviations}

ACl: Autologous chondrocyte implantation; Ad: Adenoviral; AAV: Adenoassociated virus; AD-MSCs: Adipose-derived mesenchymal stem cells; BMMSCs: Bone marrow-derived MSCs; BMP: Bone morphogenic protein; CaAb: Cartilage-targeted single-chain human antibody fragment; ECM: Extracellular matrix; ERK: Extracellular signal-regulated kinase; HIF-1a: Hypoxia inducible 
factor-1a; IGF: Insulin-like growth factor; IGF1R: Insulin-like growth factor 1 receptor; IRS: Insulin receptor-substrate; IL: Interleukin; KGN: Kartogenin; MACI: Matrix-induced ACl; MAPK: Mitogen-activated protein kinase; MMP: Matrix metalloproteinases; MSCs: Mesenchymal stem cells; mTOR: Mammalian target of rapamycin; NF-KB: Nuclear factor kappa light chain enhancer of activated B cells; NPs: Nanoparticles; OA: Osteoarthritis; PI3K: Phosphatidylinositol $3^{\prime}$-kinase; SF: Synovial fluid; OPF: Oligo (polyethylene glycol) fumarate; TGF- $\beta 1$ : Transforming growth factor- $\beta 1$; TNF-a: Tumor necrosis factor alpha; Xp-HBIGF-1: Heparin bound to IGF-1; RA: Rheumatoid arthritis; FOXO: Forkhead-box class O; SA- $\beta$-gal: Senescence-associated $\beta$-galactosidase.

\section{Acknowledgements}

Not applicable.

\section{Authors' contributions}

$C N W, L M X, X X, D P W, Y J L$, and LD participated in the literature review, figure design, and writing the review. All authors read and approved the final manuscript.

\section{Funding}

National Natural Science Foundation of China (No. 81972116; No. 81772394; No.82102607); Guangdong International Cooperation Project (No. 2021 A0505030011); Key Program of Natural Science Foundation of Guangdong Province (No.2018B0303110003); Shenzhen Science and Technology Projects (No. GJHZ20200731095606019; No. JCYJ20170817172023838; No. JCYJ20170413161649437); China Postdoctoral Science Foundation (No. 2020 M682907); Special Funds for the Construction of High-Level Hospitals in Guangdong Province.

\section{Availability of data and materials}

Not applicable.

\section{Declarations}

\section{Ethics approval and consent to participate}

Not applicable.

\section{Consent for publication}

Not applicable.

\section{Competing interests}

The authors declare that they have no competing interests.

\section{Author details}

'Department of Orthopedics, Guangdong Provincial Research Center for Artificial Intelligence and Digital Orthopedic Technology, Shenzhen Second People's Hospital, The First Affiliated Hospital of Shenzhen University, Shenzhen 518035, China. ${ }^{2}$ Department of Biomedical Engineering, Southern University of Science and Technology, Shenzhen 518055, China. ${ }^{3}$ Department of Child and Adolescent Psychiatry, Shenzhen Kangning Hospital, Shenzhen Mental Health Center, Shenzhen 518003, China.

Received: 12 February 2021 Accepted: 17 October 2021 Published online: 30 October 2021

\section{References}

1. Trippel S, et al. Gene therapy for articular cartilage repair. Proc Inst Mech Eng H. 2007;221(5):451-9.

2. Xian CJ, Foster BK. Repair of injured articular and growth plate cartilage using mesenchymal stem cells and chondrogenic gene therapy. Curr Stem Cell Res Ther. 2006;1(2):213-29.

3. van Osch GJ, et al. Cartilage repair: past and future--lessons for regenerative medicine. J Cell Mol Med. 2009;13(5):792-810.

4. Khan $\mathrm{M}$, et al. The epidemiology of failure in total knee arthroplasty: avoiding your next revision. Bone Joint J. 2016;98-B(1 Suppl A):105-12.

5. McQuillan DJ, et al. Stimulation of proteoglycan biosynthesis by serum and insulin-like growth factor-I in cultured bovine articular cartilage. Biochem J. 1986;240(2):423-30.
6. Schalkwijk J, et al. Insulin-like growth factor stimulation of chondrocyte proteoglycan synthesis by human synovial fluid. Arthritis Rheum. 1989;32(1):66-71.

7. Fukumoto T, et al. Combined effects of insulin-like growth factor-1 and transforming growth factor-beta1 on periosteal mesenchymal cells during chondrogenesis in vitro. Osteoarthritis Cartilage. 2003;11(1):55-64.

8. Wildemann $\mathrm{B}$, et al. Cell proliferation and differentiation during fracture healing are influenced by locally applied IGF-I and TGF-beta1: comparison of two proliferation markers, PCNA and BrdU. J Biomed Mater Res B Appl Biomater. 2003;65(1):150-6.

9. Madsen K, et al. Growth hormone stimulates the proliferation of cultured chondrocytes from rabbit ear and rat rib growth cartilage. Nature. 1983;304(5926):545-7.

10. Trippel SB, et al. Effect of somatomedin-C/insulin-like growth factor I and growth hormone on cultured growth plate and articular chondrocytes. Pediatr Res. 1989;25(1):76-82.

11. Longobardi L, et al. Effect of IGF-I in the chondrogenesis of bone marrow mesenchymal stem cells in the presence or absence of TGF-beta signaling. J Bone Miner Res. 2006;21(4):626-36.

12. Worster AA, et al. Chondrocytic differentiation of mesenchymal stem cells sequentially exposed to transforming growth factor-beta1 in monolayer and insulin-like growth factor-l in a three-dimensional matrix. J Orthop Res. 2001;19(4):738-49.

13. Gugjoo MB, et al. Mesenchymal stem cells with IGF-1 and TGF- beta1 in laminin gel for osteochondral defects in rabbits. Biomed Pharmacother. 2017;93:1165-74.

14. Ekenstedt KJ, et al. Effects of chronic growth hormone and insulin-like growth factor 1 deficiency on osteoarthritis severity in rat knee joints. Arthritis Rheum. 2006;54(12):3850-8.

15. Rogers SA, Hammerman MR. Insulin-like growth factor II stimulates production of inositol trisphosphate in proximal tubular basolateral membranes from canine kidney. Proc Natl Acad Sci U S A. 1988;85(11):4037-41.

16. Gelse K, et al. Articular cartilage repair by gene therapy using growth factor-producing mesenchymal cells. Arthritis Rheum. 2003;48(2):430-41.

17. Gelse K, et al. Chondrogenic differentiation of growth factor-stimulated precursor cells in cartilage repair tissue is associated with increased HIF1alpha activity. Osteoarthritis Cartilage. 2008;16(12):1457-65.

18. Starkman BG, et al. IGF-I stimulation of proteoglycan synthesis by chondrocytes requires activation of the PI 3-kinase pathway but not ERK MAPK. Biochem J. 2005;389(Pt 3):723-9.

19. Coffer PJ, Jin J, Woodgett JR. Protein kinase B (c-Akt): a multifunctional mediator of phosphatidylinositol 3-kinase activation. Biochem J. 1998;335(Pt 1):1-13.

20. Fukuda R, et al. Insulin-like growth factor 1 induces hypoxia-inducible factor 1-mediated vascular endothelial growth factor expression, which is dependent on MAP kinase and phosphatidylinositol 3-kinase signaling in colon cancer cells. J Biol Chem. 2002;277(41):38205-11.

21. Treins $C$, et al. Regulation of hypoxia-inducible factor (HIF)-1 activity and expression of HIF hydroxylases in response to insulin-like growth factor I. Mol Endocrinol. 2005;19(5):1304-17.

22. Catrina SB, et al. Hypoxia-inducible factor-1alpha and hypoxia-inducible factor-2alpha are expressed in kaposi sarcoma and modulated by insulinlike growth factor-I. Clin Cancer Res. 2006;12(15):4506-14.

23. Robins JC, et al. Hypoxia induces chondrocyte-specific gene expression in mesenchymal cells in association with transcriptional activation of Sox9. Bone. 2005;37(3):313-22.

24. Provot $\mathrm{S}$, et al. Hif-1alpha regulates differentiation of limb bud mesenchyme and joint development. J Cell Biol. 2007;177(3):451-64.

25. Malladi $P$, et al. Hypoxia inducible factor-1alpha deficiency affects chondrogenesis of adipose-derived adult stromal cells. Tissue Eng. 2007;13(6):1159-71

26. Mehana EE, Khafaga AF, El-Blehi SS. The role of matrix metalloproteinases in osteoarthritis pathogenesis: an updated review. Life Sci. 2019;234:116786

27. Chan CM, et al. Cytokine-induced MMP13 expression in human chondrocytes is dependent on activating transcription factor 3 (ATF3) regulation. J Biol Chem. 2017;292(5):1625-36.

28. Fosang AJ, Tyler JA, Hardingham TE. Effect of interleukin-1 and insulin like growth factor- 1 on the release of proteoglycan components and hyaluronan from pig articular cartilage in explant culture. Matrix. 1991:11(1):17-24. 
29. Montaseri A, et al. IGF-1 and PDGF-bb suppress IL-1 beta-induced cartilage degradation through down-regulation of NF-kappaB signaling: involvement of Src/PI-3K/AKT pathway. PLoS One. 2011;6(12):e28663.

30. Zhang M, et al. IGF-1 regulation of type II collagen and MMP-13 expression in rat endplate chondrocytes via distinct signaling pathways. Osteoarthritis Cartilage. 2009;17(1):100-6.

31. Oh CD, Chun JS. Signaling mechanisms leading to the regulation of differentiation and apoptosis of articular chondrocytes by insulin-like growth factor-1. J Biol Chem. 2003;278(38):36563-71.

32. Sah RL, et al. Differential effects of bFGF and IGF-I on matrix metabolism in calf and adult bovine cartilage explants. Arch Biochem Biophys. 1994;308(1):137-47.

33. Higgins, T.F. and B.D. Johnson, Effect of exogenous IGF-1 on chondrocyte apoptosis in a rabbit intraarticular osteotomy model. J Orthop Res, 2009;28(1):125-30.

34. Lu S, et al. Technical report: Correlation between the repair of cartilage and subchondral bone in an osteochondral defect using bilayered, biodegradable hydrogel composites. Tissue Eng Part C Methods. 2015;21(12):1216-25.

35. Zhang Z, et al. The effects of different doses of IGF-1 on cartilage and subchondral bone during the repair of full-thickness articular cartilage defects in rabbits. Osteoarthritis Cartilage. 2017;25(2):309-20.

36. Fortier LA, et al. Insulin-like growth factor-I enhances cell-based repair of articular cartilage. J Bone Joint Surg Br. 2002;84(2):276-88.

37. Ashraf $\mathrm{S}$, et al. Regulation of senescence associated signaling mechanisms in chondrocytes for cartilage tissue regeneration. Osteoarthritis Cartilage. 2016;24(2):196-205.

38. Loeser RF, Shanker G. Autocrine stimulation by insulin-like growth factor 1 and insulin-like growth factor 2 mediates chondrocyte survival in vitro. Arthritis Rheum. 2000;43(7):1552-9.

39. Loeser RF, et al. Aging and oxidative stress reduce the response of human articular chondrocytes to insulin-like growth factor 1 and osteogenic protein 1. Arthritis Rheumatol. 2014;66(8):2201-9.

40. Loeser RF, et al. Reduction in the chondrocyte response to insulin-like growth factor 1 in aging and osteoarthritis: studies in a non-human primate model of naturally occurring disease. Arthritis Rheum. 2000;43(9):2110-20.

41. Akasaki Y, et al. FoxO transcription factors support oxidative stress resistance in human chondrocytes. Arthritis Rheumatol. 2014;66(12):3349-58.

42. Zhao LD, et al. IGF-1 induces cellular senescence in rat articular chondrocytes via Akt pathway activation. Exp Ther Med. 2020;20(5):49.

43. Schalkwijk J, et al. Chondrocyte nonresponsiveness to insulin-like growth factor 1 in experimental arthritis. Arthritis Rheum. 1989;32(7):894-900.

44. Dore $S$, et al. Human osteoarthritic chondrocytes possess an increased number of insulin-like growth factor 1 binding sites but are unresponsive to its stimulation. Possible role of IGF-1-binding proteins. Arthritis Rheum. 1994;37(2):253-63.

45. Risbud MV, Sittinger M. Tissue engineering: advances in in vitro cartilage generation. Trends Biotechnol. 2002;20(8):351-6.

46. DiCarlo BB, et al. Biomaterial effects in articular cartilage tissue engineering using polyglycolic acid, a novel marine origin biomaterial, IGF-I, and TGF-beta 1. Proc Inst Mech Eng H. 2009;223(1):63-73.

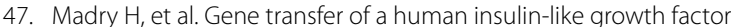
I cDNA enhances tissue engineering of cartilage. Hum Gene Ther. 2002;13(13):1621-30.

48. Madry $\mathrm{H}$, et al. Cartilage constructs engineered from chondrocytes overexpressing IGF-I improve the repair of osteochondral defects in a rabbit model. Eur Cell Mater. 2013;25:229-47.

49. Capito RM, Spector M. Collagen scaffolds for nonviral IGF-1 gene delivery in articular cartilage tissue engineering. Gene Therapy. 2007;14(9):721-32.

50. Lu S, et al. Dual growth factor delivery from bilayered, biodegradable hydrogel composites for spatially-guided osteochondral tissue repair. Biomaterials. 2014;35(31):8829-39.

51. Kim K, et al. Osteochondral tissue regeneration using a bilayered composite hydrogel with modulating dual growth factor release kinetics in a rabbit model. J Control Release. 2013;168(2):166-78.

52. O'Driscoll SW, Fitzsimmons JS. The role of periosteum in cartilage repair. Clin Orthop Relat Res. 2001;391( Suppl):S190-207.

53. Matsumoto T, et al. Differential effects of IGF-binding proteins, IGFBP-3 and IGFBP-5, on IGF-I action and binding to cell membranes of immortalized human chondrocytes. J Endocrinol. 2000;166(1):29-37.
54. Schmal H, et al. Pain perception in knees with circumscribed cartilage lesions is associated with intra-articular IGF-1 expression. Am J Sports Med. 2011;39(9):1989-96.

55. Iwanaga $\mathrm{H}$, et al. Enhanced expression of insulin-like growth factorbinding proteins in human osteoarthritic cartilage detected by immunohistochemistry and in situ hybridization. Osteoarthritis Cartilage. 2005;13(5):439-48.

56. Fraidenraich $D$, et al. Rescue of cardiac defects in id knockout embryos by injection of embryonic stem cells. Science. 2004;306(5694):247-52.

57. Jabri $\mathrm{N}$, et al. Adverse effects of recombinant human insulin-like growth factor I in obese insulin-resistant type II diabetic patients. Diabetes. 1994;43(3):369-74

58. Chan JM, et al. Plasma insulin-like growth factor-I and prostate cancer risk: a prospective study. Science. 1998;279(5350):563-6.

59. Garcia AM, et al. Transport of tissue inhibitor of metalloproteinases-1 through cartilage: contributions of fluid flow and electrical migration. J Orthop Res. 1998;16(6):734-42.

60. Madry H, Zurakowski D, Trippel SB. Overexpression of human insulin-like growth factor-I promotes new tissue formation in an ex vivo model of articular chondrocyte transplantation. Gene Ther. 2001;8(19):1443-9.

61. Orth P, et al. Transplanted articular chondrocytes co-overexpressing IGF-I and FGF-2 stimulate cartilage repair in vivo. Knee Surg Sports Traumatol Arthrosc. 2011;19(12):2119-30.

62. Hu C, et al. Introduction of hIGF-1 gene into bone marrow stromal cells and its effects on the cell's biological behaviors. Cell Transplant. 2008;17(9):1067-81.

63. Dettweiler U, Simon P. Points to consider for ethics committees in human gene therapy trials. Bioethics. 2001;15(5-6):491-500.

64. Tang, Y. and B. Wang, Gene- and stem cell-based therapeutics for cartilage regeneration and repair. Stem Cell Research \& Therapy. 2015;6(1):78.

65. Cucchiarini M, Madry H. Overexpression of human IGF-I via direct rAAVmediated gene transfer improves the early repair of articular cartilage defects in vivo. Gene Ther. 2014;21(9):811-9.

66. Kafri T. Lentivirus vectors: difficulties and hopes before clinical trials. Curr Opin Mol Ther. 2001;3(4):316-26.

67. Ortved KF, et al. Implantation of rAAV5-IGF-I transduced autologous chondrocytes improves cartilage repair in full-thickness defects in the equine model. Mol Ther. 2015;23(2):363-73.

68. Tokunou T, et al. Engineering insulin-like growth factor-1 for local delivery FASEB J. 2008;22(6):1886-93.

69. Lui JC, et al. Cartilage-targeted IGF-1 treatment to promote longitudinal bone growth. Mol Ther. 2019;27(3):673-80.

70. Bajpayee $A G$, et al. Electrostatic interactions enable rapid penetration, enhanced uptake and retention of intra-articular injected avidin in rat knee joints. J Orthop Res. 2014;32(8):1044-51.

71. Bajpayee $A G$, et al. A rabbit model demonstrates the influence of cartilage thickness on intra-articular drug delivery and retention within cartilage. J Orthop Res. 2015;33(5):660-7.

72. Geiger $\mathrm{BC}$, et al. Cartilage-penetrating nanocarriers improve delivery and efficacy of growth factor treatment of osteoarthritis. Sci Transl Med. 2018;10(469).

73. Duan $L$, et al. Exosome-mediated delivery of gene vectors for gene therapy. Nanoscale. 2021;13(3):1387-97.

74. Liang $Y$, et al. Engineering exosomes for targeted drug delivery. Theranostics. 2021;11(7):3183-95.

75. Liang Y, et al. Chondrocyte-targeted MicroRNA delivery by engineered exosomes toward a cell-free osteoarthritis therapy. ACS Appl Mater Interfaces. 2020;12(33):36938-47.

76. $\mathrm{Xu} \mathrm{X}$, et al. Exosome-mediated delivery of kartogenin for chondrogenesis of synovial fluid-derived mesenchymal stem cells and cartilage regeneration. Biomaterials. 2021;269:120539.

\section{Publisher's Note}

Springer Nature remains neutral with regard to jurisdictional claims in published maps and institutional affiliations. 